\title{
Clinical Ophthalmology
}

\section{Efficacy of phacoemulsification combined with goniosynechialysis for primary angle closure glaucoma}

\author{
I. la. Novytskyy, R. M. Lopadchak, M. I. Novytskyy
}

Danylo Halytsky Lviv National Medical University, Ophthalmology Department;

City Clinical Hospital No.8; Lviv (Ukraine)

E-mail: Inovytskyy@gmail.com

\section{Keywords:}

phacoemulsification, primary angle closure glaucoma, goniosynechialysis, IOP

\begin{abstract}
Background: To date, no universal surgical strategy exists for primary angle closure glaucoma (PACG).

Purpose: To assess the clinical efficacy and hypotensive effect of phacoemulsification (phaco) combined with goniosynechialysis (GSL) for the treatment of PACG.

Material and Methods: Nine patients (9 eyes; mean age, $63.4 \pm 9.6$ years) with chronic angle-closure glaucoma who were under our observation were included in this study. Of these, 8 (8 eyes) underwent phaco-GSL, and one (1 eye) underwent GSL at some time after phaco. Failure to control IOP (the IOP higher than $22 \mathrm{mmHg}$ on maximal tolerable hypotensive therapy) and synechial closure of the anterior chamber angle was the indication for surgery.

Results: Using the Mori goniolens was helpful in performing GSL in all anterior chamber quadrants. The IOP was $26.33 \pm 3.6 \mathrm{mmHg}$ before surgery, $18.44 \pm 0.88$ $\mathrm{mmHg}$ on day $7,18.44 \pm 1.13 \mathrm{mmHg}$ on day 30 , and $18.11 \pm 1.5 \mathrm{mmHg}$ on day 90 . Visual acuity improved from $0.09 \pm 0.05$ to $0.35 \pm 0.2$. Mean number of hypotensive medications decreased from $2.8 \pm 0.92$ pre-surgery to $0.77 \pm 0.83$ post-surgery.

Conclusion: Phacoemulsification in combination with GSL has a good hypotensive effect. Intraoperative visualization of the anterior chamber angle and transection of synechia in all quadrants allows improving the efficacy of GSL procedure.
\end{abstract}

\section{Introduction}

Primary angle closure (PAC) is present when there are features in the eye indicating that trabecular meshwork obstruction by the peripheral iris has occurred. The term glaucoma is added if glaucomatous optic neuropathy is present: primary angle closure glaucoma (PACG) [1]. Ethnic background is one of the major factors determining susceptibility to PAC. Among people aged 40 years and over, the prevalence of PAC ranges from $0.1 \%$ in Europeans through $1.4 \%$ in East Asians and up to $5 \%$ in Greenland Inuit. Of those over 40 years old in European derived populations, $0.4 \%$ are estimated to have PACG. Three quarters of cases occur in female subjects. There are 1.60 million people in Europe and 581,000 people in the USA with PACG [2].

Thick lens and short axial length are the anatomical features predisposing individuals to PACG, and may result in a pupillary block, shallow anterior chamber, and, consequently, elevated intraocular pressure (IOP) [3].

Therefore, extraction of a thick lens seems important for the management of PACG. Several studies have demonstrated that cataract phacoemulsification with intraocular lens (IOL) implantation only (phaco) could effectively reduce IOP in PACG [3, 4]. However, phaco alone fails to adequately reduce IOP in patients with chronic angle-closure glaucoma with goniosynechiae [5], and it has been proposed to use goniosynechialysis (GSL) (to break synechia) in combination with phaco in these cases $[6,7]$. Phacoemulsification with IOL plus GSL (phaco-GSL) is more effective in reducing IOP than GSL alone $[8,9]$.

The purpose of this study was to assess the clinical efficacy and hypotensive effect of phaco combined with GSL for the treatment of PACG.

\section{Material and Methods}

Nine patients ( 9 eyes; 6 women and 3 men; mean age, $63.4 \pm 9.6$ years) with chronic angle-closure glaucoma (as per the classification scheme from the European Glaucoma Society Terminology and Guidelines for Glaucoma [10]) who were under our observation were included in this study. Of these, 8 ( 8 eyes) underwent phaco-GSL, and one (1 eye) underwent GSL at some time after phaco. Failure

(C) Novytskyy I.Ia., Lopadchak R.M., Novytskyy M.I., 2020 
to control IOP (the IOP higher than $22 \mathrm{mmHg}$ on maximal tolerable hypotensive therapy) and synechial closure of the anterior chamber angle was the indication for surgery. Indentation gonioscopy showed synechial closure of the anterior chamber angle in the four quadrants in all affected eyes.

Preoperative examination included uncorrected and best-corrected visual acuity, refractometry, Maklakoff tonometry (readings obtained with the 10-g Maklakoff tonometer), anterior segment and fundus biomicroscopy, gonioscopy, indentation gonioscopy, ultrasonic ocular biometry, anterior chamber depth and lens thickness measurements, and optical coherence tomography (OCT) of the anterior chamber angle. The number of hypotensive medications used by patients was recorded, with a dualaction glaucoma drug taken into account as two singleaction drugs.

\section{Surgery technique}

A routine phaco chop procedure with a corneal tunnel incision, circular capsulorhexis and posterior chamber IOL implantation into the capsular bag was performed.

Gonioscopy was performed intraoperatively to determine whether to perform goniosynechialysis. Viscoelastic was introduced into the anterior chamber to make it deeper and to open the iridocorneal angle into the anterior chamber, especially at the sites of the anterior chamber angle. A Mori goniolens was used to visualize the anterior chamber angle. Goniosynechialysis was performed if goniosynechiae were present. To this end, a spatula introduced through a corneal paracentesis was used to push the iris root circumferentially in an attempt to make the anterior chamber angle completely open (Fig. 1). The ability to visualize the trabeculae was evidence of success of transection of synechia (Fig. 2). Thereafter, viscoelastic was removed by irrigation/aspiration, and paracenteses were hydrated.

Postoperatively, topical broad spectrum antibiotics and topical dexamethasone eye drops were administered five times daily, and pilocarpine $1 \%$, three times daily for ten days. After postoperative day 10, dexamethasone was tapered to three times daily for 20 days. Hypotensive eye drops were withdrawn postoperatively and re-administered depending on the IOP level.

The efficacy criteria were the presence and type of peri- and post-operative complications, openness of the anterior chamber angle, IOP reduction and improvement in visual acuity. Success of glaucoma surgery was defined as IOP below $22 \mathrm{mmHg}$ (readings obtained with the 10 -g Maklakoff tonometer) with or without hypotensive therapy.

Postoperatively, examinations were performed and IOP was measured at day 1 , day 7 , month 1 and month 3 .

Ethical approval was obtained from the Ethics Committee of the Lviv National Medical University and the study adhered to the tenets of the Declaration of Helsinki. Measures to ensure safety, health, rights and dignity of patients and to adhere to ethical standards were taken when conducting the study.
Data are presented as mean and standard deviation (SD). Student's t-test was used for normally distributed variables.

\section{Results}

In one case, there was mild intraoperative hemorrhage from iris vessels during transection of synechia; this, however, did not compromise surgery. Anterior chamber hemorrhage was removed during irrigation/aspiration.

A Mori goniolens was helpful in achieving transection of synechia in all the quadrants.

On the day after surgery, all the eyes were quiet and showed no signs of inflammation. In addition, the anterior chamber was of moderate depth and, excluding the three cases that had experienced preoperative pupil paresis, the pupil was round and mobile.

On day 7 after surgery, gonioscopy showed a completely open angle in all the quadrants in 6 eyes, in 3 quadrants in 4 eyes, and in 2 quadrants in 2 eyes. Open angle of the anterior chamber was defined as one where the trabecular meshwork was visible, i.e., restored aqueous access to the trabecular meshwork. Gonioscopy data were confirmed by anterior chamber angle OCT. Anterior chamber depth increased in all operated-on eyes, the mean pre- and postoperative values being $2.39 \pm 0.27 \mathrm{~mm}$ and $3.38 \pm 0.38$ $\mathrm{mm}$, respectively. In addition, the IOP decreased to below $22 \mathrm{mmHg}$ in all operated-on eyes.

Table 1 presents mean IOP values before and at time points after surgery.

The mean number of medications decreased from $2.80 \pm 0.92$ before to $0.77 \pm 0.83$ after surgery, and the difference was significant $(\mathrm{p}<0.01)$.

BCVA improved in 6 patients and remained stable in 3 patients. The mean BCVA increased from $0.09 \pm 0.05$ to $0.35 \pm 0.20(\mathrm{p}<0.01)$.

\section{Example case}

A 57-year-old patient was admitted with a diagnosis of "grade $2 \mathrm{~b}$ open angle glaucoma OU, moderate hyperopia OU, optic nerve pit OD, and amblyopia OS" on October 25 , 2019. At presentation, UCVA OD was 0.07 and BCVA OD was 0.7 with a spherical correction of $+5.5 \mathrm{D}$. In addition, UCVA OS was hand motion and BCVA OS was 0.02 with a spherical correction of $+5.5 \mathrm{D}$. The IOP OD was 34 $\mathrm{mm} \mathrm{Hg}$ and the IOP OS was $31 \mathrm{~mm} \mathrm{Hg}$ (The patient was taking Azarga twice daily and Luxfen once daily for both eyes). On examination OU, the conjunctiva was pale pink, the cornea smooth and transparent, the anterior chamber somewhat shallow, the iris unremarkable, the pupil sluggish and round, and vitreous transparent. In addition, mild lens opacification was observed. In the fundus OD, the optic disc was somewhat pale, its margins clear, vessels of regular caliber, and redistribution of macular pigment could be seen. Gonioscopy showed closed angles in all quadrants OD (Fig. 3). Anterior chamber angle width was $12^{\circ}$ in the nasal quadrant and $15^{\circ}$ in the temporal quadrant OD (Fig. 4). Ultrasonic ocular biometry found that lens thickness was $5.1 \mathrm{~mm}$ OD. The patient was 
diagnosed with "grade 2c angle closure glaucoma, severe hyperopia OU, optic nerve pit OD, and severe amblyopia OS". He underwent phacoemulsification OD on October 30, 2019. On day 1 after surgery, UCVA OD was 0.2 , and IOP OD was normal on palpation. On day 7 after surgery, UCVA OD was 0.4 , BCVA OD was 0.7 with a cylindrical correction of $-0.75 \mathrm{D}$, and IOP OD was $24 \mathrm{mmHg}$ (the patient was taking Azarga twice daily and pilocarpine 1\% thrice daily). Gonioscopy OD showed a narrow angle of the anterior chamber, adhesion of the peripheral iris to the trabecular meshwork in the upper quadrants, partially open angle in the lower quadrants, and mildly pigmented trabecular meshwork.

The patient underwent goniosynechialysis due to poor IOP control on December 11, 2019. On day 10 after surgery, UCVA OD was 0.7 , and IOP was $19 \mathrm{mmHg}$. The patient was taking pilocarpine $1 \%$ thrice daily. At month 3 postoperatively, UCVA OD was 0.7 , and IOP was 19 $\mathrm{mmHg}$ without medication. Gonioscopy OD (Fig. 5) and OCT OD (Fig. 6) showed an open anterior chamber angle in all quadrants and mildly pigmented trabecular meshwork.

\section{Discussion}

The case presented stresses the value of gonioscopy in the diagnosis of angle closure glaucoma (the patient came to us with a diagnosis of open angle glaucoma). The main reason to distinguish PACG from primary open angle glaucoma is the initial therapeutic approach and the possible late complications (particularly, synechial closure of the chamber angle) [10]. To date, gonioscopy is the only technique that can reliably diagnose PACG. PAC becomes more likely as the separation between the iris and the trabecular meshwork decreases. The risk of iridotrabecular contact in a "narrow" angle begins to increase once the iridotrabecular angle is $\leq 20^{\circ}$. There is not a precise extent of gonioscopically evident iridotrabecular contact which will dictate the indication to treatment for all cases [11].

Clinical reports of phacoemulsification with posterior chamber lens implantation in the treatment of acute, chronic and secondary angle-closure glaucoma describe very favorable results. The appropriate role of lensectomy in the management of primary angle-closure, however, remains unproven. The hypotensive effect of phacoemulsification is the release of pupil block and anterior chamber deepening, whereas GSL opens the anterior chamber angle and restores aqueous outflow through the anterior chamber angle filtration apparatus $[4,10]$. Our example case demonstrates inadequate efficacy of phacoemulsification only for synechial angleclosure glaucoma. In this case, the patient underwent GSL soon after phacoemulsification, which resulted in normalization of the IOP. The treatment outcome of this case demonstrates that it is phacoemulsification in combination with GSL that is appropriate for synechial angle-closure glaucoma. Tian and colleagues [1] found that, in the chronic angle-closure glaucoma group, IOP decreased from $26.00 \pm 11.2 \mathrm{mmHg}$ to $14.93 \pm 2.7 \mathrm{mmHg}$ after phaco-GSL surgery. Harasymowycz and colleagues [8] found that phacoemulsification and goniosynechialysis (PEGS) decreased mean IOP by $25 \mathrm{~mm} \mathrm{Hg}$, and a more apparent hypotensive effect of the surgical procedure was associated with a high average preoperative IOP $(40.7 \mathrm{~mm}$ $\mathrm{Hg}$ ) due to acute PACG.

The advantage our approach is that we perform intraoperative synechial angle closure diagnostics after phacoemulsification. After phacoemulsification, we introduced viscoelastic into the anterior chamber to make the latter deeper, especially at the site of the anterior chamber angle. Tang and colleagues [12] used a technique for transection of synechia which is similar to ours. We used this technique for intraoperative gonioscopy and for effective GSL in the presence of synechiae.

The advantage of using the Mori goniolens is that it allows the procedure to be performed without tilting the microscope or the eye and can be performed in an upright position. What is most important is that this goniolens is helpful in performing GSL in all anterior chamber quadrants. We believe that this technique improves the efficacy of GSL procedure.

Phacoemulsification in combination with GSL has a good hypotensive effect and may be a procedure of choice for synechial angle closure glaucoma. Intraoperative visualization of the anterior chamber angle and transection of synechia in all quadrants allows improving the efficacy of GSL procedure.

\section{References}

1. Tian T, Li M, Pan Y, Cai Y, Fang Y. The effect of phacoemulsification plus goniosynechialysis in acute and chronic angle closure patients with extensive goniosynechiae. BMC Ophthalmol. 2019 Mar 4;19(1):65. doi: 10.1186/ s12886-019-1070-9.

2. Alaghband $\mathrm{P}$, Rodrigues IA, Goyal S. Phacoemulsification with Intraocular Implantation of Lens, Endocyclophotocoagulation, and EndoscopicGoniosynechialysis (PIECES): A Combined Technique for the Management of Extensive Synechial Primary Angle Closure Glaucoma. J Curr Glaucoma Pract. 2018. Jan-Apr 2018;12(1):45-9. doi: 10.5005/jp-journals-10028-1243.

3. Shrivastava A, Singh K. The effect of cataract extraction on intraocular pressure. Curr Opin Ophthalmol. 2014 Mar;25(2):122-6. doi: 10.1097/ICU.0000000000000033.

4. Liu Y,LiW, Jiu X, Lei X, Liu L, Yan C, LiX. Systematic Review and Meta-Analysis of Comparing Phacoemulsification Combined with goniosynechialysis to other mainstream procedures in treating patients with angle-closure glaucoma. Medicine (Baltimore). 2019 Oct;98(42):e17654. doi: 10.1097/MD.0000000000017654.

5. Zhang H, Tang G, Liu J. Effects of phacoemulsification combined with goniosynechialysis on primary angle-closure glaucoma. J Glaucoma. 2016 May;25(5):e499-503. doi: 10.1097/IJG.0000000000000297.

6. Lai JS, Tham CC, Chua JK. Efficacy and safety of inferior 180 degrees goniosynechialysis followed by diode laser peripheral iridoplasty in the treatment of chronic angleclosure glaucoma. J Glaucoma. 2000 Oct;9(5):388-91. doi: 10.1097/00061198-200010000-00007. 
7. Lai JS, Tham CC, Lam DS. The efficacy and safety of combined phacoemulsifica-tion, intraocular lens implantation, and limited goniosynechialysis, followed by di-ode laser peripheral iridoplasty, in the treatment of cataract and chronic angle-closure glaucoma. J Glaucoma. 2001 Aug;10(4):309-15. doi: 10.1097/00061198-20010800000011.

8. Harasymowycz PJ, Papamatheakis DG, Ahmed I, Assalian A, Lesk M, Al-Zafiri Y, et al. Phacoemulsification and goniosynechialysis in the management of unresponsive primary angle closure. J Glaucoma. 2005 Jun;14(3):186-9. doi: 10.1097/01.ijg.0000159131.38828.85.

9. Takanori K, Toshihiro I, Masaru I. Long-term efficacy of goniosynechialysis com-bined with phacoemulsification for primary angle closure. Graefes Arch Clin Exp Ophthalmol. 2013 Mar;251(3):825-30. doi: 10.1007/s00417-012-2091-8. Epub 2012 Jun 29.
10. European Glaucoma Society (EGS). Terminology and Guidelines for Glaucoma. 4th Edition. Nice, France: European Glaucoma Society (EGS), 2014. p.100-129.

11. Shwartz GF, Qulgley HA. Adherence and persistence with glaucoma therapy. Surv Ophthalmol. 2008 Nov;53 Suppl1:S57-68. doi: 10.1016/j.survophthal.2008.08.002.

12. Tang Y, Tan J, Zhou X, Li X. Modified phacoemulsification plus goniosynechialysis compared with conventional surgery for cataract and glaucoma. Exp Ther Med. 2020 Jan; 19(1): 131-6. doi: 10.3892/etm.2019.8182.

The authors certify that they have no conflicts of interest in the subject matter or materials discussed in this manuscript.

Table 1. Mean IOP values before and at time points after phacoemulsification in combination with goniosynechialysis

\begin{tabular}{|c|c|c|c|c|}
\hline \multirow{2}{*}{ Characteristic } & \multicolumn{4}{|c|}{ Before surgery and at time points after surgery } \\
\cline { 2 - 5 } & Before surgery & Day 7 & Month 1 & Month 3 \\
\hline IOP & $26.33 \pm 3.6$ & $19.44 \pm 1.13$ & $18.44 \pm 0.88$ & $18.44 \pm 1.13$ \\
\hline P & & $<0.01$ & $<0.01$ & $<0.01$ \\
\hline
\end{tabular}

Note: $\mathrm{P}$ is a reliability coefficient 

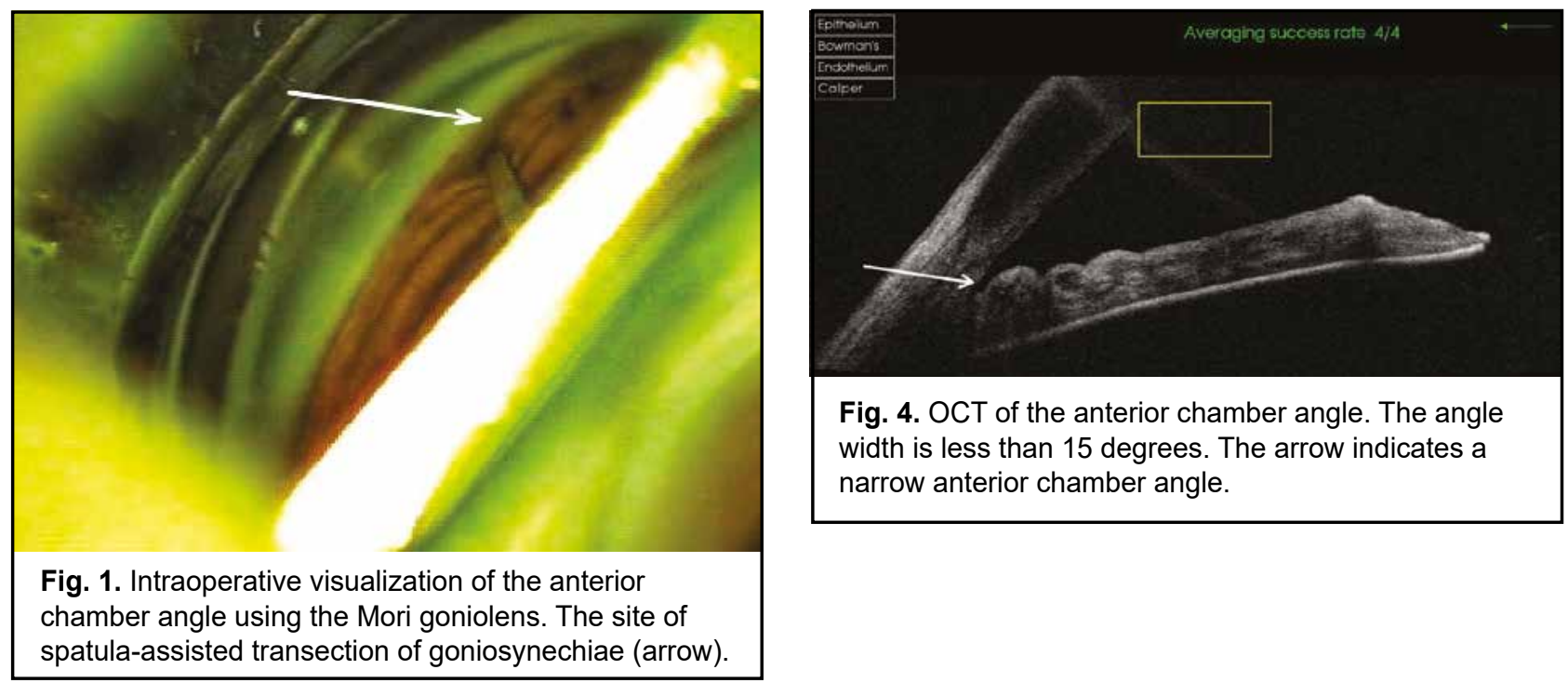

Fig. 4. OCT of the anterior chamber angle. The angle width is less than 15 degrees. The arrow indicates a narrow anterior chamber angle.
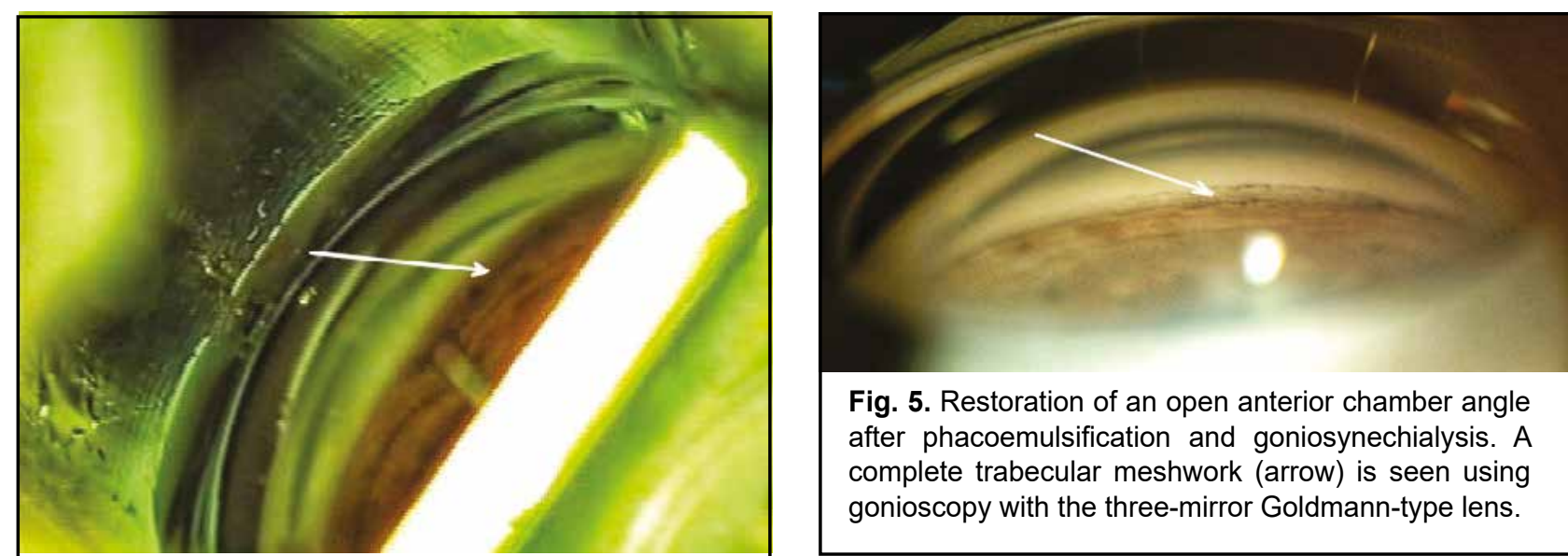

Fig. 5. Restoration of an open anterior chamber angle after phacoemulsification and goniosynechialysis. A complete trabecular meshwork (arrow) is seen using gonioscopy with the three-mirror Goldmann-type lens.

Fig. 2. Intraoperative visualization of the anterior chamber angle using the Mori goniolens. Restoration of an open anterior chamber angle (arrow) after goniosynechialysis, the trabecular meshwork is visualized with the goniolens.
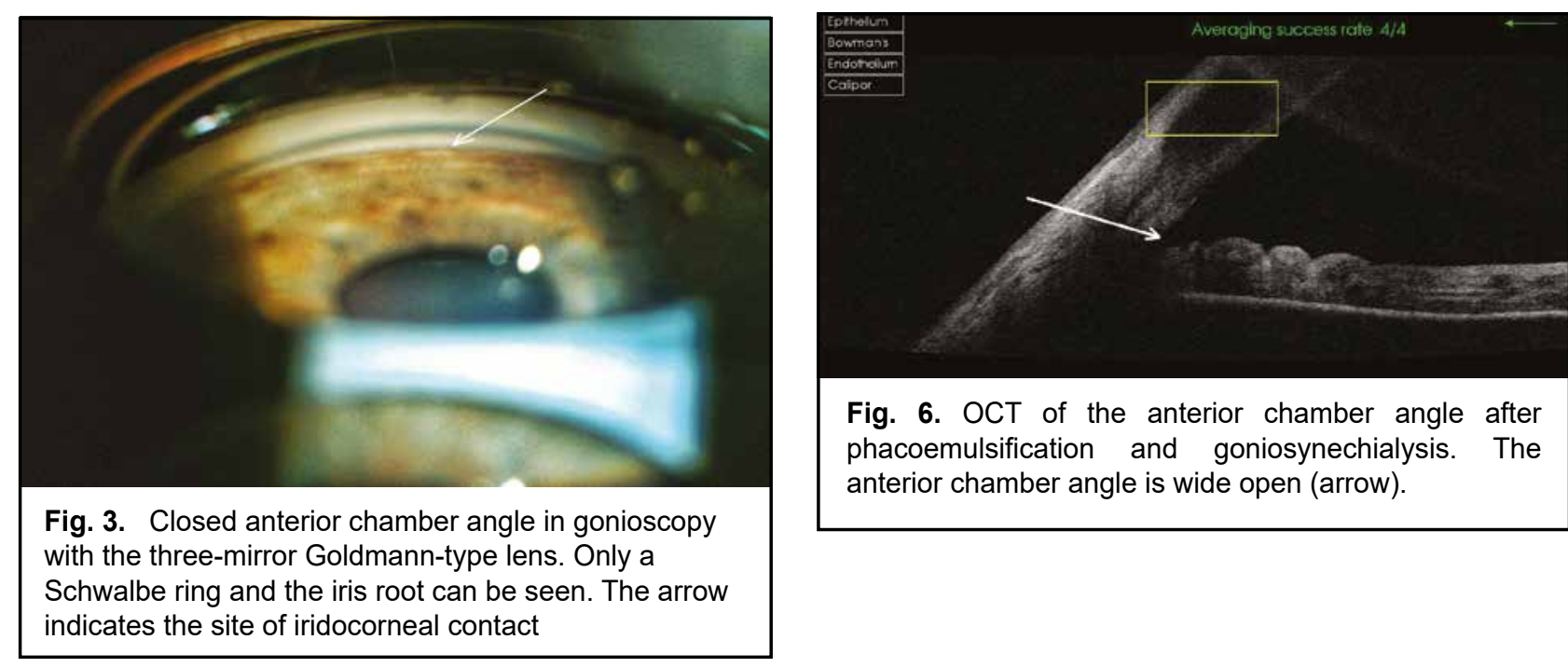

Fig. 6. OCT of the anterior chamber angle after phacoemulsification and goniosynechialysis. The anterior chamber angle is wide open (arrow). 